\title{
LIBERALISMO, PLURALISMO Y COACCIÓN
}

a idea de tolerancia es un concepto normativo de notable ambigüedad. Su función en una sociedad liberal no deja de plantear problemas sobre su fundamento y límites, ya sea vista como una medida prudencial de quienes toleran, o como un reconocimiento de los derechos de quienes son tolerados. En este trabajo utilizo los conceptos de neutralidad y daño como recursos explicativos de las actitudes tolerantes, con el objetivo de que despejen algunas de sus contradicciones y paradojas.

\section{I}

Los liberales han sido acusados de mantener una actitud de indiferencia ante las distintas concepciones de lo bueno; como ha sostenido Sandel, los liberales no establecen diferencia alguna entre el permiso y la consideración positiva de una conducta, entre la simple tolerancia y el compromiso ${ }^{1}$. Es obvio que no es lo mismo permitir la edición de material pornográfico que aprobar su contenido, como tampoco es lo mismo defender la libertad de expresión que aprobar las opiniones expresadas en el ejercicio de tal libertad. El problema consiste en determinar si del liberalismo se deriva alguna concepción ideal de la sociedad entre la pluralidad de hecho que se da en las sociedades desarrolladas, y también en precisar qué actitud toma frente a esta pluralidad de opciones. Porque parece que del escepticismo no se puede derivar lógica ni históricamente una conexión necesaria o contingente con la tolerancia. A menudo se ha considerado a la tolerancia como deudora de una actitud escéptica que impide conocer verdades religiosas y morales y que tiende a favorecer una disposición de laissez-faire

\footnotetext{
${ }^{1}$ Sandel (1984), pág. 1.
} 
frente a creencias y opiniones diferentes y contradictorias. R. Tuck ha estudiado este punto de vista y creo que ha demostrado que en la historia del escepticismo se ha defendido tanto la tolerancia como la represión ${ }^{2}$.

Tampoco la idea de la neutralidad -como se verá más adelante aporta argumentos definitivos para determinar una guía justificativa de las conductas liberales ante problemas de pluralidad de opciones. El propio concepto de autonomía puede explicar el compromiso del liberalismo con la pluralidad, la libertad y la tolerancia, pero algunas veces lo hace como un mecanismo instrumental de carácter prudencial y sólo dentro de ciertos límites.

Se tendrá que recordar una vez más que la concepción liberal se caracteriza por su compromiso con la autodeterminación individual. Lo que se valora no es la posesión de la verdad moral, sino el ejercicio de la autonomía en su búsqueda: el hecho de que los individuos sean los creadores de sus propias vidas exige una actitud tolerante. Los problemas surgen cuando se presentan individuos o grupos que no consideran a la autonomía como un valor en el orden de sus preferencias: la tolerancia se presenta entonces como un mal necesario -en virtud de las pésimas consecuencias que se podrían derivar de una actitud intolerante- y no como un bien genuino. La defensa de la tolerancia es, pues, puramente pragmática, y ésta era, precisamente, una de las dificultades conceptuales de la defensa escéptica y relativista de la tolerancia. Si el liberalismo pretende justificar la tolerancia sobre el respeto de la autonomía personal no puede llegar a este tipo de estrategias pragmáticas. Pero si la consideración de la autonomía personal es relevante, ¿qué actitud se toma, si no es la puramente pragmática, ante los grupos disidentes que cuestionan el propio valor de la autonomía?

Ante este tipo de dificultades se ha sugerido que lo que une al punto de vista liberal no es un mismo fundamento conceptual, sino una misma actitud práctica. Lo que une a los liberales se debe más a lo que son que a por qué lo son ${ }^{3}$. No les une un único fundamento identificado con algún valor singular -como podría ser la autonomía, la libertad o la igualdad- sino más bien un acuerdo pragmático acerca de las relaciones entre el Estado y los individuos. En La filosofía y el espejo de la naturaleza critica Rorty el papel central que lo que él entiende por epistemología ha tenido en la historia de la filosofía. Según Rorty, la

${ }^{2}$ R. Tuck (1988), págs. 21-35.

${ }^{3}$ Hay numerosos ejemplos que son partidarios de este punto de vista. Como muestra de ello, Waldrom (1987), pág. 144: «A liberal state is envisaged as one in which people will practice and pursue a variety of opposing and incommensurable life styles». «Liberais resolve not to favour any particular ends, or to impose on citizens a preferred way of life», Sandel (1984), pág. 3. 
epistemología parte del supuesto infundado de que todas las aportaciones a un discurso son conmensurables, es decir, pueden someterse a un conjunto de reglas para llegar a acuerdos en los puntos conflictivos. La epistemología ve a los participantes en un discurso como unidos en una universitas, un grupo unido por el interés en conseguir una finalidad común, mientras que la hermenéutica los ve unidos en una societas, en la que juntan personas unidas más por la urbanidad que por un objetivo común. Se trata de sustituir, en la terminología de Rorty, el afán de objetividad por el de solidaridad. Si la epistemología ha pretendido objetividad, lo ha hecho porque ha presupuesto la idea de que existe un lugar común descubrible mediante un ejercicio de la racionalidad. Pero, siguiendo con la interpretación de Rorty, la idea de que existe un lugar común ha ido cayendo en descrédito, lo cual ha borrado las fronteras entre verdades permanentes y contingentes: no puede decirse ya que existen en el hombre un punto central ahistórico y una periferia contingente, sino que la contingencia es la categoría central de nuestras vidas. Para Rorty la verdad y la bondad de nuestros juicios o nuestros mandatos no se vislumbran midiéndolos con referencias ahistóricas e inmutables, sino que son asuntos de práctica social, de conversación. La Ilustración sostenía que tener algo por verdadero es creer que puede afirmarse justificadamente, y busca en la razón aquella facultad que permite justificar las conductas y nuestros juicios de modo universal. El pragmático sabe, por el contrario, que parte de manera contingente de una comunidad y que sólo logrará justificar sus pretensiones ante aquellos con quienes puede mantener una conversación, es decir, ante una comunidad con la que contingentemente comparte los supuestos necesarios para conversar.

Si la pretensión pragmática resuelve el problema del pluralismo entre puntos de vista inconmensurables sin acudir a ningún criterio fundamentador -como sería, por ejemplo, el principio de autonomía-, la distinción entre la neutralidad como fundamento filosófico del liberalismo y la neutralidad como guía práctica de la concepción liberal puede servirnos para resolver algunos problemas concretos que surgen de la aplicación de la tolerancia. Porque si algo une a las pretensiones liberales es su idea directiva de que el Estado tiene que ser neutral entre las distintas concepciones de lo bueno que surgen en la sociedad. No creo que el liberalismo lo consiga, porque los límites que impone son lo suficientemente ambiguos e indeterminados como para albergar respuestas incompatibles.

El concepto de neutralidad es ambiguo, de lo cual se han derivado numerosas interpretaciones ${ }^{4}$. No es lo mismo ser neutral respecto de los resultados que de las motivaciones. No se defiende la misma idea de neutralidad entre quienes otorgan mayor o menor relevancia a las 
omisiones como posibles factores de responsabilidad moral. No es lo mismo no intervenir para no alterar el status quo que hacerlo para intentar nivelar situaciones de desigualdad. En su artículo Liberalism Dworkin sostiene:

«Political decisions must be, so far as is possible, independent of any particular conception of the good life, or of what gives value to life. Since the citizens of a society differ in their conceptions (of what the good life consists in), the government does not treat them as equals if it prefers one conception to another, either because the officials believe that one is intrinsically superior, or because one is held by the more numerous or powerful group ${ }^{5}$.

Dworkin cree en la exigibilidad de la neutralidad en virtud de su aceptación del principio de igual consideración y respeto de las personas. Existiendo consenso acerca de que el Estado pueda hacer cumplir principios de la moral «intersubjetiva» o pública, los cuales prohíben afectar ciertos intereses de individuos distintos del agente, la cuestión de la neutralidad se centra en si el Estado puede también hacer valer, a través de sanciones y otras técnicas de motivación de las conductas, pautas de la moral «personal», las cuales valoran a las acciones por sus efectos en el carácter moral del propio individuo que las ejecuta. Mientras que la posición liberal en esta cuestión consiste en decir que el Derecho no puede estar dirigido a imponer modelos de virtud personal o planes de vida (los cuales presuponen a su vez algún modelo de virtud personal), la posición opuesta sostiene que la posición del Estado consiste en hacer que los hombres se orienten correctamente hacia formas de vida virtuosa. Dworkin sostiene que ambas posiciones asignan una interpretación diferente del principio de igual consideración y respeto (todos los hombres deben ser tratados como iguales, lo que no siempre supone que todos deben ser tratados de igual modo); mientras que la primera teoría supone que las decisiones políticas deben ser independientes de cualquier concepción de lo bueno o de lo que da valor a la vida, la segunda teoría sostiene que el contenido del tratamiento igualitario no puede ser independiente de alguna concepción de lo bueno para el hombre o de lo que es bueno en la vida, ya que tratar a una persona como a un igual significa tratarla de la forma en que una persona buena y con información completa desearía ser tratada.

Si para Dworkin la igualdad es el fundamento filosófico de la concepción liberal, otros autores se refieren a principios distintos, aunque comparten en cierto sentido sus consecuencias prácticas. Dice Ackerman:

\footnotetext{
${ }^{4}$ Como muestra de ello, véase Goodin/Reeve, Liberal Neutrality (1989).

${ }^{5}$ Dworkin (1985), pág. 191.
} 
«In proposing neutrality I do not imagine I am defending an embattled citadel on the fringe of modern civilisation. Instead, I am pinting to a place well within the cultural interior that can be reached by countless pathways of argument coming from very different directions. As time passes, some paths are abandoned while others are worn smooth; yet the exciting work on the frontier cannot blind us to the hold that the center has upon us» (1980, pág. 12).

De modo que Ackerman sostiene que si bien la neutralidad es básica para la práctica de la política liberal, es indiferente el camino por el que se llega a ella. Ackerman coincide con Dworkin al afirmar la necesidad de una política neutral entre las distintas concepciones de lo bueno, pero disiente en su fundamento basado en la idea de igual consideración y respeto.

El liberalismo defiende la idea de que el Estado es un mecanismo instrumental para la satisfacción de las preferencias individuales, aunque éstas no deben considerarse simplemente como preferencias exógenas. La sociedad liberal se identifica con un escenario neutral en el que una gran variedad de modos de vida opuestos e inconmensurables persiguen sus objetivos. Evidentemente, la prohibición de interferencia estatal tiene ciertos límites, y en la moderna filosofía política se ha venido utilizando la distinción entre lo correcto y lo bueno. Lo correcto viene a determinar los límites dentro de los cuales los individuos persiguen su propia concepción de lo bueno. El concepto de lo correcto delimita el área dentro de la cual está permitida la interferencia estatal; el concepto de lo bueno delimita el ámbito de libertad en el cual no está permitida esa interferencia.

«The principles of right, and so of justice, put limits on which satisfactions have value; they impose restrictions on what are the reasonable conceptions of one's good... we can express this by saying that the concept of right is prior to that of good» (Rawls, 1971, pág. 31).

Cuando Dworkin habla de los derechos como «triunfos» frente a las posibles decisiones políticas, lo hace en este sentido. Los «derechos» delimitan el área de prohibición de la interferencia estatal -aunque ésta sea necesaria para su protección y garantía-: retiran de la agenda de las decisiones políticas un área de valores indiscutibles e innegociables. Los derechos tienen por función neutralizar preferencias externas, o sea, preferencias acerca de cómo deben vivir los demás.

Ahora bien, ¿es tan clara la línea de separación entre lo correcto y lo bueno? Se ha dicho a menudo que el lugar donde se sitúa esa línea está a su vez determinado por nuestra propia concepción de lo bueno. 
En realidad, lo correcto se determina en función de lo bueno. Esto nunca será aceptado por los liberales, para quienes lo correcto es anterior e independiente a cualquier concepción de lo bueno. Además afecta al principio de neutralidad, ya que según la crítica formulada no existen concepciones de lo bueno independientes de la interpretación que se le dé a la propia idea de neutralidad. Creo que para delimitar los problemas -e intentar resolverlos- que se derivan del intento de demarcación de estos dos ámbitos es necesario acudir de nuevo al principio del daño tal y como fue formulado por Mill.

Según Ackerman (1980, pág. 11) el principio de neutralidad liberal podría ser enunciado del siguiente modo: ningún ejercicio del poder sobre alguien puede justificarse si exige defender a quien ejerce el poder que, en primer lugar, su concepción del bien es mejor que la que tiene alguno de sus ciudadanos, o, en segundo lugar, él es intrínsecamente superior a uno o más de sus ciudadanos. De manera que lo que implica tal principio es una libertad igual para todos en virtud de la negación de los dos axiomas anteriores. No todos los liberales han defendido el principio de igual libertad y ello se ha debido a distintos argumentos críticos de este principio. Señalaré cinco.

El primer argumento rechaza el primer axioma. Viene a decir que hay concepciones de lo bueno intrínsecamente superiores a otras, y esto debe tomarlo en cuenta el Estado para romper justificadamente el principio de igual libertad ${ }^{6}$.

El segundo argumento sostiene que aunque se acepte que todos los modos de vida son igualmente válidos, no se sigue que todas las concepciones de lo bueno deban ser toleradas. Porque el primer axioma puede ser considerado, o bien como una cuestión de hecho -se constata empíricamente que existen distintas concepciones del bien- o bien como una pretensión moral. Si se trata de un enunciado de hecho, la tolerancia de estas concepciones sólo se justifica si añadimos un principio moral: que la gente que mantenga distintas concepciones de lo bueno está legitimada para

seguirlas ${ }^{7}$. El problema consistiría ahora en determinar de dónde viene este

${ }^{6}$ Por ejemplo, véase la posición de Haksar. También Raz defiende una idea perfeccionista de la libertad al considerar la libertad política personal como un aspecto de la vida buena (Raz, 1986, pág. 265). Raz sostiene que el principio de autonomía es un principio perfeccionista: la vida autónoma es valiosa sólo si se ejerce para buscar proyectos valiosos y aceptables, y el principio de autonomía permite e incluso exige a los gobiernos la creación de opciones moralmente valiosas, y eliminar las que son inmorales.

${ }^{7}$ En realidad lo que aquí se valora es el principio de autonomía, tanto si se entiende como la capacidad para llevar a cabo la elección que se ha decidido, como la propia capacidad de elegir; véase Husak (1981). 
principio moral. Si se trata de un enunciado moral -como es el principio que se ha añadidose podría manifestar del modo siguiente: la gente está legitimada para actuar según sus concepciones de lo bueno porque un sistema moral personal es tan bueno como otro. Ahora bien, ¿cómo puede alguien que defienda el principio de neutralidad argüir que un sistema moral es tan bueno como otro sin abandonar el neutralismo?

El neutralista podría sostener que el primer axioma incorpora un enunciado moral de diferente tipo: no todas las concepciones de lo bueno tienen igual mérito, pero todas las personas, en tanto que capaces de adoptar una concepción de lo bueno, merecen igual respeto. Aquí se adopta el primer axioma con el contenido del segundo: se trata de una pretensión fuerte, cuya negación implicaría una forma fuerte de perfeccionismo ${ }^{8}$.

También se ha dicho que la justificación neutralista de la libertad no es completamente neutral, ya que al admitir una concepción particular de la forma ideal de la vida humana -la que, por ejemplo, proclama el ideal de tolerancia incorpora dentro de modo implícito un elemento perfeccionista. A veces se expresa de la siguiente forma: el principio de neutralidad no puede ser neutral acerca de la neutralidad; o lo que es lo mismo, no se puede tolerar a los intolerantes. El principio de neutralidad, explicitado en los dos axiomas, no puede tratar neutralmente ideas perfeccionistas, racistas o sexistas que nieguen cualquiera de los dos axiomas. Del mismo modo que, justamente como demócratas, no deberíamos ser «demócratas» con respecto a la democracia, tampoco como neutralistas deberíamos ser neutrales respecto de la neutralidad. El principio de neutralidad no justifica cualquier libertad, sino que más bien distingue entre libertades legítimas e ilegítimas.

Sin embargo, una implicación de la intolerancia de los intolerantes, es que favorece los estilos de vida individualistas sobre los comunitarios. Es decir, como los grupos amenazan la libertad de sus miembros para seguir sus propias concepciones del bien, el Estado neutral tiene que intervenir e

${ }^{8}$ Se pueden distinguir dos tipos de perfeccionismo (Farrell, 1991): el perfeccionismo débil mantiene solamente que una vida autónoma es una parte esencial del bienestar humano; desde este punto de vista, las consideraciones perfeccionistas se reducen a sostener que algunas formas de vida son más idóneas que otras para determinadas personas. Para caracterizar al perfeccionismo fuerte, Farrell utiliza la distinción de Barry (1978, págs. 38-39) entre principios want-regarding y principios ideal-regarding. Los primeros toman como algo dado las preferencias de la gente, y se preocupan en determinar los efectos que las medidas políticas tienen sobre la satisfacción total de las preferencias, así como de la distribución de oportunidades para su satisfacción. Por el contrario, los principios ideal-regarding no sólo defienden el valor de la autonomía, sino que también deciden qué deseos merecen ser satisfechos. 
interferir tales grupos para proteger las elecciones personales individuales. Esto unido al hecho de que los modos de vida individualistas son menos costosos al exigir una menor cooperación, hace que se promocionen este tipo de planes de vida frente a los grupales o societarios. Precisamente esta es la crítica que Raz formula a Rawls (Raz, 1986): el Estado puede ser neutral solamente si crea las condiciones de iguales oportunidades para elegir cualquier concepción del bien, con una posibilidad o perspectiva igual de llevarla a cabo. Esto implica la intervención estatal para proteger los modos de vida amenazados (sean individualistas o colectivistas) de extinción. No obstante, este tipo de interferencia niveladora está llena de deficultades: ¿Qué implica la promoción igual de las concepciones de lo bueno? ¿Cómo se pueden medir las perspectivas para llevar a cabo una concepción de lo bueno? ¿Cuándo se puede decir que estas perspectivas son iguales? Más adelante volveré sobre este problema.

Por último, el neutralismo no puede tratar neutralmente los conflictos entre concepciones de lo bueno excluyentes entre sí. El principio de neutralidad protege la libertad de cada persona para seguir su concepto de lo bueno sólo hasta que no se amenace la misma libertad de alguien para perseguir su bien. Entonces, ¿cómo puede el principio de neutralidad tratar situaciones en la que una concepción del bien puede sólo ser perseguida a costa de otra concepción del bien?

El neutralista no debe tolerar la imposición de preferencia externas. No debe tolerar concepciones dañinas del bien: se debe ser neutral entre los amantes de la ópera y los del rock, pero no entre los amantes de la ópera y los violadores. Hay un área de inmunidad en la que funcionan los derechos más que los bienes o lo bueno; la neutralidad requiere un principio adicional -como por ejemplo, el principio del daño- para justificar la libertad.

II

La cuestión acerca de si las normas jurídicas deben imponer coactivamente, es decir, con su aparato de fuerza, ciertas convicciones morales ha sido un problema muy debatido desde los comienzos de la modernidad. La filosofía de los límites del poder tiene su origen histórico en una mentalidad que se genera en Europa durante las guerras de religión, y se manifiesta teóricamente en la distinción entre el iustum y el honestum, o lo que es lo mismo, en la distinción kantiana posterior entre el foro «interno» $\mathrm{y}$ «externo» como criterio de división de las obligaciones por su ámbito de aplicación ${ }^{9}$. Estas distinciones conceptuales son la consecuencia de dos siglos de persecuciones religiosas y

\footnotetext{
${ }^{9}$ Sobre la filosofía de los límites del poder como precedente de la filosofía de los derechos humanos, veáse Peces Barba (1982).
} 
de intromisiones legales en el ámbito de la conciencia individual. La libertad religiosa expresaba la pretensión de la libertad individual frente al poder estatal, la prohibición moral de someter la conciencia individual a normas jurídicas. La filosofía de los límites del ejercicio del poder exigía la distinción entre dos tipos de obligaciones: las morales y las jurídicas.

«Se dice entonces que mientras la obligación moral vincula territorios del fuero interno y extrae su fundamento y mérito del motivo interno del obrar, la obligación jurídica somete tan sólo la exterioridad de la acción y exige sólo el cumplimiento externo del mandato sea cual sea el motivo de ese cumplimiento: cálculo, temor, prudencia, etc... Y es precisamente por esa ignorancia de los motivos del obrar por lo que puede incorporar la «coacción» sin disminuir un ápice el valor jurídico del cumplimiento. Algo que, sin embargo, es impensable en la ponderación del valor moral del cumplimiento de las obligaciones éticas que, si se «fuerza», pierde toda su virtud, pero es muy importante que no perdamos de vista el sentido último de todo este movimiento ético-político porque en realidad no tiene la vista puesta en privar al derecho y al poder de un fundamento moral, sino muy por el contrario en formular precisamente un nuevo fundamento moral para el estado político: sólo es legítimo aquel sistema de gobierno que no trata de regular la conciencia individual» (Laporta, 1990, págs. 255 y 256).

Esta tradición encuentra en el principio de Mill formulado en On Liberty su plasmación teórica de un modo muy sencillo:

«Este principio es que el único fin para el que el género humano está autorizado, individual o colectivamente, a interferir en la libertad de acción de cualquiera de sus miembros es la propia protección. Que el único propósito con el que el poder puede ser legítimamente (rightfully) ejercido sobre cualquier miembro de una comunidad civilizada contra su voluntad es para prevenir el daño a otros. No puede ser legítimamente compelido a hacer u omitir algo porque ello sea mejor para él, porque le vaya a hacer más feliz, porque, en la opinión de otros, hacerlo fuera sabio o incluso moralmente correcto».

De modo que la moralidad de una acción no es razón suficiente para que esa acción pueda ser impuesta por la fuerza, ni tampoco su inmoralidad lo es para imponer su prohibición de modo coactivo. Sólo el evitar un daño a otros puede funcionar como justificación de la 
imposición coactiva de una conducta. La opinión moral de la mayoría no es fundamento suficiente para dar contenido a las normas jurídicas, ni lo es tampoco el bienestar o la felicidad de los destinatarios. En contra del primer postulado se encuentra la posición del moralismo legal; en contra del segundo, el paternalismo. También el perfeccionismo ignora estos postulados: sostiene que lo que es bueno para un individuo o lo que satisface sus intereses es independiente de sus propios deseos o de su elección de forma de vida, teniendo el Estado, mediante distintos medios, la potestad de dar preferencia a aquellos intereses y planes de vida que son objetivamente mejores.

Hay autores que han señalado la compatibilidad del liberalismo con enfoques objetivistas y perfeccionistas acerca de la apreciación de intereses. Se ha dicho, por ejemplo, que mientras las teorías negativas de la libertad subrayan la posibilidad de hacer lo que se quiere sin la existencia de obstáculos externos, las teorías positivas asumen que la libertad consiste en algún tipo de realización personal que no depende de los deseos del agente. A veces nos equivocamos en la apreciación de nuestros intereses -nuestro proceso de formación de nuestras preferencias se ve interferido por disonancias cognitivas-, y a veces las restricciones a nuestras acciones no deben ser vistas como limitaciones a nuestra libertad. Quien ha defendido recientemente no sólo la compatibilidad entre perfeccionismo y liberalismo sino su implicación necesaria ha sido $\mathrm{V}$. Haksar ${ }^{10}$. Para este autor sólo si se asume que hay formas de vida superiores a otras se puede afirmar que hay algo que tienen en común todos los hombres por lo que deben ser acreedores de igual consideración y respeto. La posición perfeccionista afirma que la vida humana es intrínsecamente valiosa por contar con la capacidad de proponerse y desarrollar de modo autónomo planes de vida. Esta posición tiene presupuestos pragmáticos, que justifican no hacer diferencia entre los hombres respecto del grado en que desarrollan su autonomía. Según Haksar la concepción perfeccionista lleva a valorar como mejores los planes de vida que expanden la autonomía de los individuos: esto implica que otros planes de vida tienen un status inferior en la sociedad liberal, aunque de aquí no se sigue que los individuos que los ejecutan sean inferiores o merezcan menos respeto. Tampoco se deben prohibir los planes de vida que se consideren inferiores porque esto iría contra el derecho a igual respeto de cada uno. Pero el Estado puede abstenerse de facilitar y financiar planes de vida degradantes, del mismo modo que puede promocionar planes de vida meritorios así como proyectos que protegen el bienestar de futuras

${ }^{10}$ V. Haksar (1979). 
generaciones. Como ha sostenido Nino ${ }^{11}$, Haksar propone un compromiso entre el desaliento de las formas inferiores de vida y la tolerancia de quienes las siguen.

También Raz ha sostenido un punto de vista similar ${ }^{12}$ 12.Raz sostiene que un sistema moral basado en el valor de la autonomía no puede tener como elementos primitivos derechos individuales, ya que la autonomía requiere bienes colectivos que no pueden ser el contenido de derechos individuales puesto que no son objeto de deberes por parte de otros. Añade que es imposible ser neutral acerca de ideales de lo bueno, ni tampoco se puede excluirlos como razones para la acción política. Precisamente, una razón poderosa por la que no se puede mantener consistentemente una postura de neutralidad viene dada por el hecho de que los ideales personales de la moral autorreferente pueden expandirse hacia aspectos de la organización social que implican una invasión del ámbito de lo correcto. Además, el bienestar de los individuos está intrínsecamente unido a formas de organización social, por lo que la división tajante entre moral intersubjetiva y moral personal -que le sirve a Mill para formular su principio del daño a terceros- se diluye necesariamente. Asimismo, las concepciones de lo bueno basadas en la satisfacción de las preferencias individuales -como parecen defender las concepciones subjetivistas del liberalismo- no pueden separarse de los valores que subyacen a ellas, es decir, de la validez de las razones que las determinan. El criterio objetivista exige discriminar entre las razones que están detrás de las preferencias, estableciendo una jerarquización de esas razones según que sean centrales o periféricas para la vida humana ${ }^{13}$. Precisamente, Raz considera que los bienes colectivos como la tolerancia y el respeto son bienes públicos para la sociedad porque son bienes para sus miembros. Lo mismo cabe decir de la cultura pública y la tolerancia religiosa.

Nino distingue entre concepciones de lo bueno y planes personales de vida. Si bien el liberalismo descansa indudablemente en una concepción de lo bueno -o de lo que es socialmente bueno- según la

${ }^{11}$ Nino (1989), pág. 207. El propio Nino ha criticado los enfoques objetivistas y perfeccionistas, aduciendo que no sólo es muy difícil hacer explícito cuáles son los planes de vida o intereses favorecidos y descalificados de acuerdo con presuntos criterios objetivos, sino que tampoco se ven claros cuáles son los límites de la intervención estatal en favor de los planes de vida e intereses privilegiados: su discrepancia con el perfeccionismo radica en si la evaluación positiva o negativa de los distintos modos de vida -evaluación cuya posibilidad se comparte- debe tener relevancia jurídica.

${ }^{12}$ Raz (1986), págs. 198 y sigs. Véase también Raz (1989b).

${ }^{13}$ Sobre la concepción liberal de los derechos y sus límites, puede verse Prieto (1990). 
cual la autonomía de los individuos para elegir y materializar proyectos y estilos de vida es intrínsecamente valiosa, de esto no se sigue que el Estado deba adoptar una preferencia por ciertos planes de vida sobre otros. Al contrario, la preferencia por algún plan de vida es incompatible con la concepción de la autonomía como intrínsecamente valiosa, ya que la idea de «preferencia» incluye alguna idea de interferencia en la elección de planes de vida. Para Nino la idea de que la vida autónoma sea una parte esencial del bienestar humano no es una especie de perfeccionismo ya que la autonomía no es una propiedad de algunos planes de vida, sino la capacidad para elegir entre la más amplia variedad posible de ellos.

El liberalismo debe ser compatible con una concepción objetivista del bienestar de acuerdo con la cual las preferencias dependen de creencias en ciertos valores. Esto en principio es incompatible con la idea de que sólo se puede garantizar la autonomía personal si se parte de una concepción subjetivista de lo bueno; si lo que es bueno para los individuos es algo objetivamente determinable parece que favorece la idea de su posible imposición con independencia de las decisiones y preferencias individuales. Pero creo que es distinto mantener una concepción objetiva de lo bueno y mantener o justificar su vinculatoriedad coactiva. Una cosa es justificar el contenido de una pretensión y otra bien distinta justificar su imposición coactiva. Del mismo modo, puedo tener razones para pensar en la legitimidad de la vinculatoriedad de una pretensión aunque no esté de acuerdo con su contenido. Creo que en esto radica la justificación de los sistemas políticos democráticos: son depositarios justificados de la fuerza aunque con ello no se zanja la justicia del contenido de sus decisiones.

La concepción subjetivista del bien -y, por tanto, la concepción de que las preferencias son variables exógenas inmodificables- ha sido avalada, con independencia de su identificación con el placer en las versiones más toscas del utilitarismo, por una confusión entre el enfoque interno y el externo de las preferencias ${ }^{14}$. Si preguntamos a alguien si

${ }^{14}$ Nino (1989), págs. 212 y sigs., y Nino (1989b), págs. 37 y sigs. El aspecto interno y externo de la preferencias es una versión del problema general del punto de vista interno sobre la conducta humana. Precisamente la paradoja de la tolerancia no es más que una manifestación de la tensión entre el aspecto interno y externo del discurso moral. La paradoja consiste en el hecho de que los principios avalados por los criterios de validación internos del discurso pueden llevar a permitir comportamientos que socavan las condiciones externas para que ese discurso cumpla sus propósitos (se tolera a los enemigos de la libertad). Y viceversa, hay comportamientos que pretenden justificarse sobre la base de la necesidad de crear un ámbito de libertad y democracia, y que por su carácter discriminatorio no son sin embargo admisibles, a pesar de su posible eficacia para el fin perseguido, según los criterios de validación internos de nuestro discurso (no se tolera a quien puede hacer posible el ejercicio de la libertad, pero emplea medios discriminatorios). 
considera valioso satisfacer sus propios deseos, contestará afirmativamente, no porque tenga esos deseos sino porque valora las cosas que respaldan esos deseos, y considera que es valioso satisfacer lo que considera que es valioso. Esto implica que para satisfacer deseos de otros se tiene que tener en cuenta no el hecho de que tengan tales deseos, sino la validez de las razones que los determinan. Nino sostiene que el desconocimiento del aspecto interno de las preferencias al asignar valor a su simple satisfacción tiene una consecuencia muy negativa: parece quitar todo valor a la autonomía. Si la libertad consiste en la satisfacción simple de las preferencias o deseos individuales, y si no hay ningún valor que preceda la formación de los deseos, entonces parece que la satisfacción de las preferencias reveladas no puede tener valor. Pero si se tiene en cuenta el aspecto interno de las preferencias y se las satisface sólo en la medida de la validez de las razones que las respaldan, esto parece facilitar la imposición coactiva de ciertos valores con independencia de las preferencias reveladas. Lo que hace disminuir la intensidad de la autonomía.

Ahora bien, si la autonomía se entiende más como un valor objetivo que forma una parte esencial de cualquier concepción del bien, toda acción que en nombre del bien perjudique tal autonomía es autofrustrante. No se puede llevar a cabo la imposición coactiva de ciertos valores transgrediendo un componente esencial del contenido de cualquier concepción del bien.

Pero volvamos al principio del daño a terceros como guía legislativa de la concepción liberal. Según Mill es el daño a terceros la única garantía de la interferencia estatal: los gobiernos tienen que tolerar aquellas acciones que no caen bajo su ámbito. Pero ¿cuál es el criterio en virtud del cual se puede decir que una conducta causa daño? En su personal interpretación de Mill, J. Gray dice:

«Does he intend the reader to understand 'harm' to refer only to physical harm, or must a class of moral harms to character be included in any apllication of the liberty principle? Must the harm that the restriction of liberty prevents be done to identifiable individuals, or may it also relevandy be done to institutions, social practices and forms of life? Can serious offence to feelings count as harm so far as the restriction of liberty is concerned...? Can a failure to benefit someone, or to perform one's obligations to the public, be construed as a case in which harm is done?» (Gray, 1983, pág, 49).

La interpretación de todos estos elementos hace que la versión del concepto de «daño» pueda variar profundamente. El principio del daño establece un límite negativo que no puede traspasar las acciones de los sujetos autónomos. Este límite tiene que enfrentarse con el problema de su puesta en práctica para solucionar los posibles conflictos que surjan entre las exigencias de la justicia y las del ejercicio de la autonomía. 
El principio del daño de Mill es un ejemplo del principio de neutralidad. En este sentido, sostiene Raz:

«The doctrine of political neutrality claims that (some) political actions should be neutral regarding ideals of the good life, that implementation or promotion of ideals of the good life is, though worthy in itself, not a legitimate ground for (some) political actions. Such a doctrine is a doctrine of restraint since (as understood here) it advocates neutrality between valid and invalid ideals of the good alike. It demands not only that the promotion of unacceptable ideals should not be the ground for (some) political actions, but also that the promotion of acceptable, correct, desirable ideals should be equally shunned» (Raz, 1982, pág. 91).

Es decir, mientras que el principio del daño permite algunas veces la interferencia legal, la imposición de un ideal nunca justifica tal intervención. Ahora bien, ¿se puede trazar una clara distinción entre acciones que causan daño y acciones que provocan simplemente cierto tipo de disgusto, aversión o desaprobación? ¿Las acciones que provocan desaprobación o disgusto son en sí mismo dañinas?

En el ámbito anglosajón el Report of the Commitee on Obscenity and Film Censorhip (The Williams Committee) de 1979 apelaba directamente a Mill al sostener que «ninguna conducta debería suprimirse por el Derecho a menos que se demostrara que produce daño a alguien». El concepto de «daño» que utiliza el Comité es el daño físico real, y éste es un criterio demasiado estricto como para que pueda llevarse hasta sus últimas consecuencias. Pero si el concepto de «daño» se construye de manera amplia, abarcando aspectos tan ambiguos como el daño moral, la ofensa o la simple molestia o impertinencia, cualquier conducta, dado el hecho del pluralismo de preferencias y deseos, podría ser penalizada bajo el amparo de este criterio tan impreciso. De modo que si el concepto de daño se construye de una manera restrictiva, se dejan al margen del Derecho conductas sobre las que sería necesario cierto amparo legal; y si se construye amplio, puede justificar la interferencia legal de modo ilimitado. Además, nuestras discrepancias sobre los contenidos del concepto de «daño» no se deben a simples estipulaciones lingüísticas, sino a una divergencia de nuestras más profundas convicciones morales ${ }^{15}$.

${ }^{15}$ A veces nos molesta el simple conocimiento de la existencia de modos de vida y opiniones que consideramos inmorales. ¿Estaría incluida esta simple incomodidad en el contenido del principio del daño formulado por Mill? Waldrom ha contestado de modo convincente (Waldrom, 1987) a esta cuestión. En el trabajo citado sostiene que la respuesta no puede ser resuelta mediante un análisis del concepto de daño, sino en virtud de los argumentos que se defienden en On Liberty. Por consiguiente, como Mill 
Con referencia a las tesis del Comité Williams, Dworkin señala lo siguiente:

«Suppose «harm» is taken to exclude mental distress, but to include damage to the social and cultural environment. Then the harm condition is in itself no help in considering the problem of pornography, because opponents of pornography argue, with some force, that free trafic in obscenity does damage the general cultural environment» (Dworkin, 1985, pág. 337).

Dworkin sostiene que el principio del «daño» no ofrece ningún criterio o guía para justificar o deslegitimar la acción estatal. De modo que ofrece un concepto alternativo basado en la idea de «derechos», el cual le sirve para diseñar una estrategia que delimite el área de intervención estatal. No obstante, Dworkin sostiene que aunque no hay un derecho general a la libertad, ciertas libertades tienen el status de derechos -en un sentido fuerteporque están justificadas por el principio de «igual consideración y respeto». Este principio es una justificación de ciertas libertades ya que otras pueden ser restringidas para promocionar el interés público sin impugnar el principio de igual consideración y respeto.

III

La formulación de Dworkin de la posición liberal puede ser expresada en términos de neutralidad, aunque este término no sea precisamente un ejemplo de claridad conceptual. No obstante puede proporcionar las pistas de la relación de la tolerancia con la idea del pluralismo y el principio del daño ${ }^{16}$.

El concepto de neutralidad presupone una disputa o conflicto entre dos o más partes (personas, equipos, naciones, religiones, ideales, valores), y centra su atención en una parte adicional, o tercera parte, cuyas acciones y status son propiamente el objetivo de la calificación de neutralidad o no neutralidad. En este sentido se puede decir que la imagen de la tríada judicial (demandante, defensor, juez) se invoca a menudo, pero no siempre la tercera parte necesita actuar como mediadora para ser juzgada como neutral o no neutral; a veces, si esta tercera parte se ocupa de sus propios asuntos ignorando el conflicto puede ser calificada de neutral.

valoraba positivamente la confrontación de opiniones morales como medio del progreso moral, también debía valorar como un bien positivo -más que como un daño- el simple disgusto o molestia aludida.

${ }^{16}$ Sobre este punto mi exposición sigue la argumentación de Waldrom (1989, págs. 61-84). 
Si el presupuesto de la neutralidad es un conflicto entre dos o más partes, esto implica a su vez el hecho del pluralismo normativo. La posibilidad de lograr un consenso moral en una sociedad liberal es improbable, ya que no existe un conjunto de virtudes que sea exhaustivo o totalizador. El rechazo del pluralismo ha generado intolerancia y normalmente ha ido acompañado de prácticas políticas totalitarias. Además, algunas virtudes implican la ausencia de otras y algunas igualmente valiosas son incompatibles.

El pluralismo moral es el punto de vista que sostiene que existen distintos tipos de concepciones de vida que ejemplifican distintas virtudes incompatibles. Una concepción o plan de vida incluye nuestros propósitos, los objetivos y valores que guían nuestras acciones, así como nuestros gustos y otras preferencias. En la concepción liberal una concepción individual de la vida buena es un plan de vida o estrategia para vivir que un individuo usa como base de sus más importantes decisiones, así como para diseñar sus pretensiones e identificar sus obstáculos. No se trata de un plan de actuación detallado y fijo que pudiera responder a la pregunta ¿qué he hecho? o ¿qué haré?, sino que responde a ¿qué es lo que estoy haciendo con mi vida? Si bien mi plan o concepción de vida puede cambiar -no tan a menudo como a veces se pretende- sí tiene sentido que guarda cierta unidad reflexiva y valorativa como elemento integrador de un pasado, un presente y un futuro. Porque mi concepción de la vida buena no se puede explicar en términos puramente prudenciales: no es simplemente el ejercicio de una planificación de mis actividades para maximizar en el curso de nuestras vidas la satisfacción de las preferencias a las que profeso cierta adhesión; creo que es un asunto más reflexivo que lo que es una simple estrategia de actuación. Implica un proceso de distanciamiento crítico y compromiso con el entorno normativo en el que se vive, un proceso abierto de evaluación crítica.

Como el proceso de formación de una concepción de la vida buena implica hacer una evaluación, el legislador se ve tentado a interferir este proceso. Supongamos que millones de españoles se vean motivados por deseos de bestialismo y pedofilia y otros gustos similares; la neutralidad liberal sostiene que el legislador no debería interferir este proceso de toma de decisiones, no porque considere valioso el contenido de las mismas, sino porque cree que hay algo más importante: la propia capacidad individual para formular la evaluación de su propia vida, lo cual tiene una importancia moral independiente del valor moral del contenido de cada decisión. O lo que es lo mismo, la interferencia legal con el propósito de modificar tales evaluaciones posee un disvalor superior al que tiene el contenido de las evaluaciones decididas de modo autónomo por los sujetos sobre los que pesa tal interferencia. Precisamente, 
la teoría que explica por qué es importante y positivo el que los individuos formulen sus propias evaluaciones y por qué la interferencia legal también lo es, pero de manera negativa (con independencia del mérito moral de su intención) constituye el núcleo central de la justificación liberal de la neutralidad y la tolerancia.

Decía antes que uno de los elementos centrales para poder practicar la tolerancia es el pluralismo. El ejercicio de la autonomía individual se lleva a cabo mediante la elección entre distintas opciones, que dada la limitación de nuestros recursos, se presentan a veces como incompatibles. La incompatibilidad no desmerece el valor de cada opción, el cual está determinado por las razones que justifican cada una de ellas. Si la autonomía es considerada como intrínsecamente valiosa, esto conduce a decir que tener más opciones es más valioso que tener menos, juicio al que se adhieren los economistas. Elegir entre distintas opciones es bueno porque, o bien contribuye a obtener otras cosas buenas o bien se considera que es un bien en sí mismo. Al aumentar las opciones se contribuye a aumentar el bienestar de los individuos porque se incrementa la probabilidad de que uno pueda ver cumplidos sus deseos. Aparte de este valor instrumental se dice que la posibilidad de elegir contribuye positivamente a la formación o autorrealización moral de las personas. En este sentido se sostiene que lo que tiene valor intrínseco no es disponer de distintas opciones, sino ser consciente de la capacidad que uno tiene para elegir entre distintas alternativas. Esto fundamenta la capacidad de elegir, el hecho de tener algunas opciones, pero no el tener más opciones. No siempre el tener más opciones beneficia la satisfacción de los intereses individuales, pues es bien conocido el proceso de formación de preferencias distorsionadas, tal y como vimos anteriormente.

Tener en cuenta esto contribuye a aclarar una errónea identificación entre dos ámbitos distintos, en los cuales si bien los procesos de formación de las preferencias son similares, no debe ser así su toma en consideración. Porque esto último puede generar distintos modos de comprender la política que se lleva a cabo en un sistema democrático. No es lo mismo, como hacen los partidarios de la teoría de la elección social, compartir una concepción del proceso político como un mecanismo instrumental en el que los actos decisivos son los votos secretos -es decir, actos privados-, y en el que el propósito de la política es el compromiso óptimo entre intereses privados exteriorizados, opuestos e irreductiblemente inconmensurables, que, por el contrario, sostener que la conducta fundamental de los procesos políticos es pública, y que la naturaleza de la política no es instrumental. Según, por ejemplo, Habermas, el propósito de la política sería más bien el acuerdo racional que el simple compromiso; los actos políticos decisivos no serían tanto 
los constitutivos de los votos, sino la discusión en debates públicos con el objetivo de lograr un consenso. Según los teóricos de la democracia participativa -como por ejemplo, J. S. Mill- el propósito de la política es la transformación y educación de los participantes: la política es un fin en sí mismo, y su cuestión central es más bien la transformación de las preferencias que la simple agregación.

De acuerdo con esta concepción, el núcleo del proceso político es el análisis público y racional acerca del bien común, y no el acto aislado de votar de acuerdo con preferencias privadas. La meta de la política debe llegar a conseguir el consenso unánime y racional y no un compromiso óptimo entre intereses inconmensurables. En definitiva, el foro no debe verse contaminado por los principios que regulan el mercado ${ }^{17}$.

Volvamos de nuevo a la imagen de la neutralidad. Decía antes que el concepto de neutralidad presupone un conflicto entre dos o más partes, y también la existencia de una tercera parte cuya conducta es el referente de la existencia o no de una conducta neutral. Dos aspectos deben señalarse: en primer lugar, que la conducta de la tercera parte no puede ser descrita como neutral si toma partido en el debate que se discute; en segundo lugar, si las acciones o existencia de la tercera parte no pueden influir en el debate, tanto en su curso como en los resultados, entonces no surge la cuestión de la neutralidad.

Este concepto ha encontrado en el Derecho Internacional un campo de abono, pero incluso en este ámbito no ha tenido un significado fijo e incontrovertible. Propondré un ejemplo: un gobierno neutral tiene el deber de abstenerse en la ayuda a cualquiera de dos partes beligerantes; pero no se exige la prohibición a sus ciudadanos privados de negociar con alguno de los dos países beligerantes. De modo que hay una distinción implícita entre acción gubernamental y negocios privados, lo cual en principio sirve como línea de demarcación entre acciones neutrales y no neutrales. Pero, ¿qué sucede si el Estado controla la actividad económica de su sociedad directa o indirectamente mediante empresas nacionalizadas o por algún otro mecanismo de economía intervenida?

El concepto de neutralidad es impreciso y depende del contexto en el que se utilice; no es lo mismo su uso en la diplomacia internacional que como ideal judicial; también es distinta la idea de neutralidad cuando se habla de colores, gustos o elementos químicos, la neutralidad en el análisis metaético, en el ámbito de las ciencias sociales, en la

${ }^{17} \mathrm{~J}$. Elster, The market and the forum: three varietes of political theory, en Elster/Hylland (1989), págs. 103-132. 
educación, etc. Así, cuando el liberalismo habla de la deseabilidad de una legislación moralmente neutral, no está claro a qué tipo de concepción se refiere ${ }^{18}$.

Por ejemplo, cuando Dworkin sostiene que un legislador tiene que ser neutral entre distintas concepciones competitivas de lo bueno, ¿está hablando acerca de la neutralidad respecto de la intención del legislador, o acerca de la neutralidad de los efectos de las acciones del legislador? Hay autores, como por ejemplo Raz (1986) o Montefiore (1975), que han interpretado la neutralidad liberal con respecto a las consecuencias de la acción legislativa: un Derecho neutral no puede aumentar las oportunidades de llevar a cabo un peculiar estilo de vida en detrimento de otro, ni tampoco, aumentar las probabilidades de elegir un plan de vida en detrimento de otro. Este criterio presenta grandes dificultades, porque es muy difícil encontrar el punto de referencia con el que se tiene que medir los efectos de la legislación. En lugar de ello, los liberales pueden hablar de neutralidad de intenciones, es decir, neutralidad respecto de los motivos y razones que usa el legislador para justificar las leyes: el poder no debe ejercerse en virtud de razones no neutrales. Por ejemplo, si el Estado prohíbe trabajar los sábados no es debido a un respeto discriminatorio de la religión judía -lo cual sería una razón no neutral, sino por razones del descanso laboral (aunque se beneficie la religión judía y no otras religiones).

Como la neutralidad depende de las razones que se tienen para el establecimiento de la medida que se discute, habrá que determinar las razones que existen detrás de cada medida legal, lo que implica una gran dificultad. En realidad, no se puede decidir simplemente qué tipo de neutralidad se quiere adoptar ya que ello depende del tipo de justificación que se use en favor de la neutralidad. ¿Se basa en un compromiso positivo con la pluralidad ética? ¿Se basa en el escepticismo moral? ¿Se basa en una fe en el progreso moral? ¿Se basa en la preocupación por un posible desarrollo del perfeccionismo moral? ¿O en la creencia en el principio de igual consideración y respeto?

Por ejemplo, el argumento del escepticismo moral puede justificar una concepción de la neutralidad basada en las intenciones del agente, mientras que el argumento pluralista puede congeniar mejor con la neutralidad consecuencia lista. Porque el escepticismo moral ve como algo irracional intentar actuar con razones morales, mientras que el pluralismo moral se preocupa de que ninguna medida legislativa disminuya la pluralidad de los distintos planes de vida. En definitiva, las

${ }^{18}$ Véanse los usos del concepto de neutralidad en los diferentes ámbitos -estatal, legal, económico, educativo, científico, etc.- en el libro colectivo de Goodin y Reeve (1989). 
diferentes líneas de argumento de la posición liberal generarán diferentes concepciones de la neutralidad, las cuales generarán a su vez diferentes y quizá exigencias mutuamente incompatibles en el nivel de la práctica legislativa. Esto contradice radicalmente la tesis de Ackerman quien sostiene que debemos ser neutrales no solamente acerca de los ideales éticos, sino también acerca de la propia neutralidad ${ }^{19}$. Esto plantea una paradoja sobre la que luego volveré.

Una teoría de la neutralidad y de la tolerancia no sólo tiene que explicar por qué se exige, sino también a quién se exige y sobre qué se exige. Si bien se insiste en que el legislador tiene que ser neutral respecto de lo que constituye la vida buena, no se insiste con respecto a la gente en general. Si el concepto de vida buena tiene un uso, la neutralidad no puede ser exigida de todo el mundo, ya que lo bueno es un concepto discriminador y evaluativo.

La exigencia de neutralidad funciona mejor en el ámbito de la moralidad política: no es erróneo para alguien favorecer una concepción particular de la vida buena, pero lo es en su capacidad de legislador, de funcionario público. Creo que la neutralidad es una virtud política, porque hay algo en la vida política que la hace especialmente importante: la fuerza, la coerción, la capacidad de violencia institucionalizada, elemento que está ausente en la esfera moral o en la búsqueda de propósitos morales ${ }^{20}$.

La fuerza y la coerción son inútiles en el ámbito de la moral, porque la adhesión de una persona a una concepción de la vida buena es asunto interno de su propia conciencia. Esto lo vio muy bien Locke, para quien el uso de las sanciones en la esfera moral no tiene ningún sentido. La acción no neutral por el legislador es simplemente irracional, ya que se usan medios que no están adaptados ni son eficaces para el fin que se pretende perseguir. Por el contrario, la organización autónoma de la vida personal tiene un significado relevante. Según esto, lo erróneo de la coerción asociada al Derecho es que usurpa e interfiere el proceso significativo de la organización autónoma de la propia vida, moldeando de modo heterónomo las concepciones personales; parece que esto es inapropiado. Ahora bien, es difícil establecer con precisión la línea que demarca una conducta coercitiva de otro tipo de conducta, como por ejemplo la persuasiva. La identificación de esta línea no es un asunto estipulativo, sino que hay que preguntarse por qué importa la coerción y qué valores se esconden detrás de una práctica coercitiva.

${ }^{19}$ Ackerman, B. A. (1980). Sigo en este apartado la crítica que formula Waldrom en Goodin, R. E. y Reeve, A. (1989), págs. 61-83.

${ }^{20}$ Me refiero al ámbito de la argumentación moral, y no al de la moral institucionalizada, la cual emplea el principio de autoridad en la imposición coactiva de sus contenidos. 
Decía antes que una teoría de la neutralidad tiene que preguntarse sobre qué se exige ser neutral. La neutralidad liberal no lo es relación con todos los valores morales, ni tampoco sostiene que la legislación esté «libre de valores». Parece que estas ideas son incoherentes, ya que la neutralidad es un valor en sí misma: es una posición normativa, una doctrina acerca de lo que los legisladores y la administración debe hacer. Se podría decir que es una doctrina que sostiene que es equivocado para ciertos temas entrar en la arena política.

MacCormick ha distinguido entre teorías morales acerca de la legislación, del desarrollo y aplicación de los valores morales y mediante la legislación ${ }^{21}$. La pregunta que se hace este autor es la siguiente: ¿Hasta qué punto, sí cabe, debería utilizarse la ley para la consecución de valores morales? Porque esta es una cuestión de moralidad política, dado que se refiere al correcto ejercicio de los poderes públicos con que están investidos los órganos del Estado: el legislativo, el gobierno, jueces, policía, fiscales. El punto a discutir no es si se debería utilizar el poder del Estado en conformidad con principios morales, sino qué principios morales deberían observarse en el ejercicio del poder del Estado. Sobre ello se pueden formular dos puntos de vista, según se mantenga o no la idea de que el responsable correspondiente esté moralmente obligado a ejercer sus poderes legislativos sin hacer referencia a la cuestión de cuál es la conducta moral correcta o equivocada de los ciudadanos. Tanto si se adopta un punto de vista como el otro se está afirmando un principio moral, el que sostiene que el poder debe o no debe utilizar sus facultades para implantar valores morales. Expondré, siguiendo a MacCormick en este punto, algún argumento que justifique la no intervención estatal en este ámbito, aunque después se vea la imposibilidad de mantener la abstención estatal en materias morales, especialmente mediante la defensa del principio del daño.

Los defensores de que el Estado no deba imponer ningún contenido moral mediante sus disposiciones, subrayan la importancia de la moralidad para los seres humanos: demasiado importante como para dejarla en manos del Estado. Las exigencias de la moralidad incumben a cada ser humano en tanto que ser autónomo. Los verdaderos valores morales se llevan a cabo al margen de la coacción, por lo que la virtud moral no está constituida por una conformidad aparente hacia modelos de conducta externamente impuestos, respaldados por la amenaza de castigos legales; está constituida por el libre autocompromiso con modelos de conducta y valores internamente aceptados, y por decisiones motivadas por este autocompromiso. La disciplina moral es autodisciplina, no la

\footnotetext{
${ }^{21}$ MacCormick (1982), págs. 27-43.
} 
disciplina de la policía, tribunales y prisiones. De modo que el cultivo de los valores morales exige libertad de elección, libertad de expresión y discusión como caminos para llegar a la verdad moral.

Ahora bien, este principio está formulado en términos negativos. ¿Cuáles serían las bases permisibles para el ejercicio del poder público? La concepción liberal ha respondido con el principio del daño a otros: los poderes del Estado pueden utilizarse legítimamente para impedir que unas personas dañen a otras. Algunos, como por ejemplo Hart ${ }^{22}$, extienden el concepto de daño de manera que incluya la perpetración de ofensas a los sentimientos o sensibilidad de las personas mediante exhibiciones o manifestaciones públicas, así como ciertos paternalismos admisibles. Lo que parece obvio es que el castigo conlleva un daño producido intencionadamente a las personas mediante acciones tales como el embargo de la propiedad, o la privación de libertad, o la imposición de sufrimiento físico. El castigo es utilizado por el Estado sólo para disminuir o restringir la imposición de formas de sufrimiento similares a personas inocentes. De modo que si se defiende la idea de que el Estado no debe imponer ningún contenido moral, se hace porque se tiende a asegurar la minimización del sufrimiento humano deliberadamente producido por otros seres humanos: al cumplir el principio del daño, se permite que el Estado amenace o imponga daños por medio del castigo, pero sólo para impedir la perpetración privada de daños mayores de tipo fundamentalmente similar.

Ahora bien, la ley penal, al prohibir la conducta intencionadamente lesiva y al someter a castigos a los que producen el daño, parece hacer cumplir forzosamente exigencias que también son exigencias morales. Es decir, si el principio del daño autoriza la utilización del poder del Estado para hacer cumplir ciertos valores morales -como sería la autonomía de las personas-, esto contradice la tesis que sostiene la ausencia de un contenido moral en las disposiciones legales.

Una posible respuesta podría sostener que las leyes autorizadas por el principio del daño sólo coinciden o cubren exigencias morales aunque en realidad no hacen cumplir tales valores morales. Lo que importa a las autoridades del Estado es la calidad de dañina de la conducta lesiva, no su carácter inmoral. Pero esta interpretación estaría sujeta a dos objeciones radicales: la primera viene a decir que la justificación del principio de la ausencia de contenido moral unido con el principio del daño depende de un recurso al valor moral del respeto a las personas en tanto que agentes morales autónomos. De manera que existe un valor derivado de proteger a las personas frente a invasiones

\footnotetext{
${ }^{22}$ Hart, H. L. A. (1963) y (1965).
} 
de su autonomía, y esto expresa el contenido de un valor moral fundamental. Es frecuente encontrar en la mayor parte de las interpretaciones del principio del daño una referencia al principio de autonomía. Raz, por ejemplo, interpreta la autonomía de un modo perfeccionista, y trata de conciliar esta pretensión con el principio del daño ${ }^{23}$. Esta pretensión plantea muchas dificultades: existe una difícil compatibilidad ente el principio del daño y el ideal perfeccionista de autonomía, ya que es muy difícil evitar la interferencia de las acciones moralmente repugnantes que no producen daño a otros ${ }^{24}$.

No hay que olvidar que el Derecho penal versa en gran parte sobre cuestiones referentes a valores morales. Los delitos se definen en virtud de consideraciones normativas acerca de la justicia de la propiedad privada, la tributación o el alcance y límite de los derechos de bienestar. El recurso a la ley penal es siempre parasitario -o, al menos, subordinado- a un orden legal establecido de derechos y deberes en las esferas públicas y privadas; dicho orden ha de fundamentarse en alguna concepción sobre una ordenación justa de la sociedad.

Además, el acto de «castigar» es en sí mismo un acto expresivo y simbólico de una actitud de grave desaprobación de lo hecho por la persona castigada, sobre la base de que es considerada culpable por ello. Al hacerlo, se pone de manifiesto su desaprobación más o menos severa: expresa la condenación del Estado del acto cometido, y esto es, evidentemente, una actitud moral. De modo que cualquier principio que permita que el Estado pueda recurrir al castigo necesariamente permite la implantación estatal de algunos valores morales. Pero no sólo la ley penal refleja cierto consenso moral, sino que también tiende a generarlo: a causa de su fuerza simbólica como moralidad pública del Estado, la ley penal con su puesta en escena y simbolismo público de juicios y castigo puede, en cierta medida,

${ }^{23}$ Raz, 3. (1986). Una reciente interpretación no perfeccionista del principio de autonomía puede verse en Sadurski (1990); para este autor la autonomía es intrínsecamente valiosa, es decir, tener más opciones es más valioso que tener menos. Para Raz la autonomía no es valiosa por sí misma, sino sólo en la medida que se utiliza para la búsqueda del bien moral.

${ }^{24}$ Es cierto que el propio Raz sostiene que aunque la autonomía es valiosa sólo si persigue el bien moral, debería protegerse incluso si se usa para fines moralmente repugnantes, en virtud de consideraciones consecuencialistas, como por ejemplo el hecho de que los efectos indiscriminados de la restricción coercitiva conducen no sólo a restringir las elecciones repugnantes, sino todas sus otras opciones, aunque sean moralmente correctas. No obstante, es difícil compatibilizar la idea de autonomía de Raz con sus pretensiones perfeccionistas. 
ser constitutiva de una moralidad común para el conjunto de ciudadanos como tal ${ }^{25}$.

De manera que ante la pregunta ¿sostiene el liberalismo el principio moral de que la legislación nunca debería ser usada para imponer coactivamente valores morales?, la respuesta parece negativa, ya que los liberales consideran la neutralidad no solamente como un valor al que los legisladores deberían someterse, sino también un valor que deberían imponer sobre la gente que intenta ejercer el poder de una manera no neutral: el legislador no es neutral acerca de la neutralidad ${ }^{26}$.

Si esto es así, se puede derivar una consecuencia importante: la doctrina de la neutralidad liberal no puede ser justificada coherentemente por una apelación general al escepticismo moral. Si el no-cognitivismo emotivista es una tesis acerca del lenguaje evaluativo, el neutralismo liberal es una posición normativa y evaluativa: guía la conducta legislativa y evalúa los resultados o propósitos legislativos. Así, si el emotivismo es correcto, sostendrá que el neutralismo liberal -como concepción particular de la vida buena- es una simple cuestión de emociones; ofrece, por consiguiente, una razón para no imponer la doctrina liberal de la neutralidad; es decir, ofrece una razón para no imponer coactivamente el punto de vista de que las concepciones de la vida buena no deben ser impuestas coactivamente. De manera que la tolerancia y la neutralidad liberal no pueden justificarse sobre la base del escepticismo. La cuestión metaética es independiente del aspecto sustantivo del liberalismo.

Muy pocos liberales han defendido el escepticismo moral. La mayoría de ellos han defendido ciertos valores objetivos. Donde Hobbes fue más escéptico -en la fe y el culto religioso- fue menos liberal. De manera que el legislador liberal ha de ser sensible a ciertas discriminaciones entre valores y principios sobre las que no se puede mantener una actitud neutral. El principio de neutralidad o el valor de la tolerancia podrían ser los ejemplos más obvios. La lista podría extenderse a los «bienes primarios» que ha defendido Rawls: salud, integridad física, autorrespeto, bienestar, libertad negativa, educación... Precisamente el legislador tiene la obligación de diseñar una estructura de principios e instituciones para apoyar y distribuir estos bienes. No

${ }^{25}$ MacCormick, autor a quien he seguido en este punto, propone como alternativa al principio de ausencia de contenido moral el principio de una fundamentación moral limitada: los poderes del Estado pueden y deben ejercerse para implantar exigencias morales, pero únicamente las que son deberes de respeto a las personas que conciernen a los demás, y sólo lo mínimamente necesario para asegurar para todos las condiciones de respeto propio en tanto que seres autónomos (págs. 39 y sigs.).

${ }^{26}$ Este tipo de paradojas, como antes se ha explicado en la nota 14 , se pueden explicar recurriendo a los aspectos interno y externo del discurso moral. 
obstante, hay otros bienes que deberían quedar fuera del ámbito de la legislación: en la teoría de la tolerancia de Locke, el Estado debía abstenerse de intervenir en la salvación individual. En formulaciones más modernas -como la de Mill- la sociedad debería permanecer neutral y tolerar los diferentes estilos de vida de los individuos. Hoy se habla de "concepciones individuales de la vida buena», o de "concepciones individuales de lo bueno», esto es, de creencias individuales acerca de lo que da significado a la vida, o lo que para la vida de una persona tiene significado.

No obstante, se podría plantear alguna objeción a la concepción individualista de la vida buena. Se podría decir que aunque el liberalismo sostiene una concepción individualista, es obvio que reconoce que las personas adquieren sus gustos, valores y propósitos, y los modifican, en interacción con otras personas. La cuestión es por qué la heteronomía del legislador moralista difiere de la heteronomía moral que surge durante la vida humana en los distintos modos con que los seres humanos pueden afectar y manipular de manera deliberada los valores de otro. Además, como cuestión de hecho, no todos los puntos de vista éticos sostenidos por los individuos son concepciones individualistas de la vida buena del tipo que se han descrito. Hay otras concepciones orientadas hacia la comunidad que, en principio, exigen el mismo trato liberal que las concepciones orientadas hacia el individuo. Después de todo, como ha sostenido D. Parfit ${ }^{27}$, ¿por qué se piensa que son importantes las series inter-temporales de experiencias que constituyen para nosotros la vida individual, como opuestas a las series intertemporales y simultáneas de las experiencias que constituyen la vida de un grupo o de una sociedad?

La dificultad consiste en que las razones que los liberales ofrecen para censurar las actividades grupales de disciplina, proselitismo y coerción son razones no neutrales, diseñadas hacia una teoría individualista de la autorrealización personal. Precisamente ésta fue la crítica que Nagel dirigió a Rawls ${ }^{28}$ : los principios liberales de su conocida teoría de la justicia incorporan la situación de elección social que él mismo concibe, y la clase de bienes que define, todo ello orientado a la búsqueda de planes de vida individuales. Lejos de ser neutral, la idea liberal presupone un compromiso con una concepción individualista de la bondad, lo cual tiene efectos no-neutrales sobre las concepciones del bien que dependan de lazos sociales o grupales. Por ello se dice que la concepción liberal de la neutralidad es fraudulenta, ya que muchas

\footnotetext{
${ }^{27}$ D. Parfit (1984).

${ }^{28}$ Th. Nagel en N. Daniels (ed.) (1975), págs. 9-10.
} 
concepciones de la vida buena presuponen una estructura de relaciones políticas, económicas y sociales de tipo comunitario. Y ya que los liberales están comprometidos a no tolerar la determinación de las estructuras sociales por ideales éticos o religiosos, estas concepciones deberían quedar fuera de su ámbito, de modo que la doctrina liberal de la neutralidad es inconsistente y autofrustrante.

Ahora bien, parece que esta acusación es injustificada en virtud de las siguientes consideraciones. En primer lugar, la neutralidad como política nunca es autojustificativa, es decir, se es siempre neutral en un conflicto particular por una razón particular, de modo que no se puede ser neutral acerca de la fuerza de esa razón. Además, una política de neutralidad en relación con un conflicto no compromete a ser neutral en todos los conflictos posibles, ni tampoco en los conflictos en los que alguna de las partes participe. Si aplicamos estas consideraciones a la doctrina de la neutralidad liberal, lo único que se puede derivar es que la teoría liberal, como cualquier teoría normativa, tiene restricciones: es obvio que excluye los puntos de vista religiosos de Jomeini, pero esto es razonable. El liberalismo no ha decidido arbitrariamente qué concepción de la vida buena quiere defender; piensa que el diseño y la realización personal de un plan de vida es algo bueno. Por eso teme que este proceso se distorsione por una coerción aplicada externamente, aunque se trate de una coerción basada en la moral social de una comunidad. Precisamente las concepciones holistas o grupales del bien no son de la clase que los liberales defienden: las razones que les persuaden y convencen para ser neutrales entre las distintas concepciones del bien, también les persuaden para no ser neutrales entre las concepciones individualistas y comunitaristas del bien. Se es siempre neutral en una disputa particular por una razón particular, y de aquí no se deriva que esta consideración ofrezca una razón para ser neutral en un conflicto diferente.

\section{BIBLIOGRAFÍA}

La Bibliografía que se presenta es más extensa que la utilizada para el presente trabajo, pero al versar sobre tolerancia puede ser útil su consulta.

Ackerman, B. (1980): Social Justice in the Liberal State, New Haven \& London. Barry, B. (1978): Political Argument, Londres. 
Bellamy, R. (1989): Defining liberalism: neutralist, ethical or political?, en Liberalism and recent legal and social philosophy, ARSP, núm. 36.

Bollinger, L. C. (1986): The tolerant society, New York, OUP.

Camps, V. (1990): Virtudes públicas. Espasa-Calpe, Madrid.

Crick, B. (1971): Toleration, Government and Opposition, 6:2, págs. 144-171.

Dworkin, G. (1988): The theory and practice of autonomy, Cambridge Univ. Press.

Dworkin, R. (1985): A matter of principle, Harvard Univ. Press.

Dworkin, R. (1986): Law's Empire, Cambridge.

Dworkin, R. (1983): «What liberalism isn't, New York Review of Books, enero.

Dworkin, R. (1977): Taking Rights Seriously, Duckworth, Londres.

Elster, J. (1979): Ulysses and the Sirens, Cambridge Univ. Press.

Elster, J. (1983): Sour Grapes, C.U.P., 1983. (Hay trad. cast. Uvas amargas, Península, 1988).

Elster, J. (1986): The market and the forum, en Ester/Hylland (eds.), C.U.P.

Farrell, M. D. (1991): Autonomy and Paternalism: The Political Philosophy of J. $R a z$, RATIO IURIS, vol. 4, núm. 1, marzo 1991.

Garzón, E. (1986): Los deberes positivos generales y su fundamentación, DOXA núm. 3, Alicante.

Goodin/Reeve (1989): Liberalism and neutrality, en Goodin/Reeve, Liberal Neutrality.

Goodin/Reeve (eds.) (1989): Liberal Neutrality, Routledge, Londres.

Gray, J. (1983): Mill on Liberty: A Defence, Routledge \& Kegan Paul, Londres.

Griffin, J. (1990): Against the taste model, en Interpersonal Comparisons of Well-Being, Cambridge Univ. Press.

Griffin, J. (1990): Interpersonal Comparisons of Wellbeing, Cambridge Univ. Press.

Gross, F. (1985): Toleration and Pluralism, II Político, junio, 50:2, págs.181-219.

Haksar, V (1979): Equality, Liberty and Perfectionism. Clarendon Press, Oxford.

Harrison, G. (1976): Relativism and Tolerance. Ethics 86, págs. 122-135.

Hart, H. L. A. (1963): Law, Liberty and Morality, Londres.

Hart, H. L. A. (1965): The Morality of the Criminal Law, Londres. 
Horton/Mendus (eds.) (1985): Aspects of Toleration, Methuen, Londres.

Kelsen, H. (1982): ¿Qué es justicia?, Ariel ed.

King, P. (1971): The Problem of Tolerance, Government and Opposition, 6:2, págs. 172-207.

Laporta, F. (1989): Ética y Derecho, en Historia de la Ética, ed. Crítica.

Larmore, Ch. (1990): Political Liberalism, Political Theory, vol. 18, núm. 3.

Oxford.

Locke, J. (1968): A Letter on Toleration, Klibansky, ed., trad. de J. W Gough,

Locke, J. (1960): Two Treatises on Government, Laslett (ed.), Cambridge.

MacCormick (1982): Legal Right and Social Democracy, O.U.P.

MacIntyre, A. (1987): Tras la virtud, Crítica.

McClure, K. M. (1990): Difference, Diversity and the limits of toleration, Political Theory, vol. 18 , núm. 3 .

Mendus/Edwards (eds.) (1987): On Toleration, Oxford Clarendon Press.

Mendus, S. (ed.) (1988): Justufying Toleration: Conceptual and Historical Perspectives, Cambridge University Press.

Mendus, S. (1988): Toleration and the limits of liberalism, MacMillan.

Miller, D. (1987): Enciclopedia del Pensamiento Político, Alianza ed., 1989.

Mills, J. S. (1970): Sobre la libertad, Alianza ed., Madrid.

Montefiore (1975): Neutrality and Impartiality, Cambridge Univ. Press.

Nagel, T. (1986): A View from Nowhere, Oxford.

Nagel, T. (1990): Conflicto moral y legitimidad política, en Derecho y moral. Ensayos analiticos, Ariel.

Nicholson, P. (1985): Toleration as a moral ideal, en J. Horton y S. Mendus eds., Aspects of Toleration, Methuen, Londres.

Nino, C. S. (1989): Ética y derechos humanos, Ariel.

Madrid.

Nino, C. S. (1990): El constructivismo ético, Centro de Estudios Constitucionales,

Parfit, D. (1986): Reasons and Persons, Oxford, Clarendon Press.

Peces-Barba, G. (1982): Tránsito a la modernidad y derechos fundamentales, Mezquita, Madrid.

Popper, K. R. (1967): La sociedad abierta y sus enemigos, Paidós, Buenos Aires. 
Prieto Sanchis, L. (1990): Estudios sobre derechos fundamentales, Debate, Madrid. Raphael, D. D. (1988): The Intolerable, en S. Mendus (1988).

Rawls, J. (1985): Justice as Fairness: Political not Metaphysical, Philosophy and Public Affaires, vol. 14, núm. 3.

Rawls, J. (1987): The idea of an overlapping consensus, Oxford Journal of Legal Studies, vol. 7, núm. 1 (hay trad. cast. La idea un consenso por superposición, en Derecho y moral. Ensayos analíticos/, Ariel.

Raz, J. (1986): The Morality of Freedom, O.U.P.

Raz, J. (1990): Facing Diversity: The Case of Epistemic abstinence. Philosophy and Public Affaires, winter, vol. 19, núm. 1, págs. 3-46.

Raz, J. (1988): Autonomy, Toleration and the Harm Principle, en S. Mendus (ed.) 1988.

Raz, J. (1989): Liberalism, Skepticism and Democracy, Iowa Law Review, vol. 74, núm. 4, págs. 761-786.

Raz, J. (1982): Liberalism, Autonomy and the Politics of the Neutral Concern, Mid-West Studies in Philosophy, 7, págs. 89-120.

Richards, D. A. J. (1986): Toleration and the Constitution, New York, Oxford University Press.

Richards, D. A. J. (1988): Toleration and Free Speech, Philosophy and Public Affairs, 17:4, págs. 323-336.

Rorty, R. (1983): La filosofía y el espejo de la naturaleza, ed. Cátedra.

Sadaba, J. (1989): La filosofía moral analitica de Wittgenstein a Tugendhat, Mondadori. Publishers.

Sadurski, W (1990): Moral Pluralism and legal neutrality, Kluwer Academic

Sandel, M. (1982): Liberalism and the limits of Justice, Cambridge Univ. Press.

Sandel, M. (1984): Liberalism and its critics, Basil Blackwell, Oxford, Sandel (ed.).

Savater, E (1900): La tolerancia, institución pública y virtud privada, Claves, Madrid, núm. 5.

Scruton, R. (1982): A Dictionary of Political Thought, Londres.

Sunstein, C. (1986): Legal Interference with Private Preferences, The Univ. of Chicago Law Review, vol. 53, núm. 4.

Tinder, G. (1976): Tolerance, Toward a new Civility, Univ. of Massachusetts.

Tuck, R. (1988): Scepticism and Toleration in the Seventeenth Century, en S. Mendus (ed.), 1988. 
Tullock, G. (1971): The logic of the Law, New York.

Van de Veer, D. (1986): Paternalistic Intervention, Princeton Univ. Press.

Waldrom, J. (1987): Theoretical Foundations of Liberalism, Philosophical Quarterly, 37, 147.

Waldrom, J. (1987): Mill and the value of moral distress, Political Studies, 35, págs. 410-23.

Walzer, M. (1990): The Communitarian Critique of Liberalism, Political Theory, vol. 18 , núm. 1.

Walzer, M. (1983): Spheres of Justice, New York.

Warnock, M. (1987): The limits of Toleration, en S. Mendus y D. Edwards (eds.) On Toleration, O.U.P.

Williams, B. (1973): Problems of the Self, Cambridge Univ. Press.

Wolff, Moore, Marcuse (1969): A Critique of Pure Tolerance, Boston.

Wolff, R. P. (1970): In Defence of Anarchism, New York.

Wolff, R. P. (1968): The Poverty of Liberalism, New York. 